\title{
Presacral Myelolipoma A Case Report and Literature Review
}

\author{
CESARE GAGLIARDO, GIORGIA FALANGA, RAFFAELLO SUTERA, GIUSEPPE LA TONA, \\ ANTONIO LO CASTO, MASSIMO MIDIRI, ROBERTO LAGALLA \\ Department of Biopathology and Medical and Forensic Biotechnologies, Section of Radiological Sciences, University \\ of Palermo; Italy
}

Key words: presacral myelolipoma, presacral masses, MRI and CT findings

SUMMARY - Many reports have described adrenal myelolipomas but there have been only a few reports of extra-adrenal myelolipomas. We describe a 74-year-old woman who came to our observation for MRI of the lumbar spine for typical lumbar back pain. In addition to signs of mild scoliosis and spondylo disc arthrosis, MR imaging revealed a presacral mass showing a heterogeneously high signal in all pulse sequences and almost completely suppressed on inversion recovery sequences for fat tissue. CT imaging confirmed the fatty nature of the lesion and no signs of bone involvement. These findings were most consistent with a diagnosis of a rare presacral myelolipoma as confirmed at histopathologic analysis. This work reports a case of one of the rarest presacral masses, emphasizing the role of imaging in the differential diagnosis of other presacral masses.

\section{Introduction}

Adrenal myelolipomas are rare benign, usually small and asymptomatic, tumours characterized by mature adipose tissue and variable amounts of hematopoietic elements discovered incidentally on imaging studies. There have been only few reports of extra-adrenal myelolipomas. Hence we describe a case of presacral myelolipoma presenting as an incidental finding in an elderly woman with aspecific lower back pain signs and symptoms.

\section{Case Report}

We describe a 74-year-old woman who came to our attention for an MRI of the lumbar spine. The patient complained of lumbar back pain characterized by low back stiffness aggravated by rest with pain centred in the hips, buttocks and thighs, aggravated by hyperextension with positive straight leg raise test with typical irradiation to the lower limbs as L4-L5-S1 radiculopathy. No significant signs of sacral root involvement were found; defecation and urination were almost unremarkable.
MRI of the lumbar spine, performed with a $1.5 \mathrm{~T}$ scanner (Achieva Philips Medical Systems; Cleveland, OH, USA) revealed signs of mild scoliosis (at least in a non-weight-bearing examination) associated with widespread signs of spondylo and disc arthrosis with robust marginal osteophytes. All of these findings were compatible with the reported symptoms and clinical signs. MRI examination also revealed a presacral oval multilobed mass with smooth edges and thin interlobar septa and heterogeneously high signal in all sequences but almost completely suppressed on inversion recovery sequences for fat tissue (T2w Short Tau Inversion Recovery, STIR; T1w Spectral Presaturation Inversion Recovery, SPIR) with several solid nodules with intermediate signal in all sequences. The day after, a CT scan was scheduled and MRI examination was performed after IV administration of paramagnetic contrast.

CT examination, performed with a 16-channel scanner (BrightSpeed CT 16 GE Medical System; Milwaukee, WI, USA), confirmed the fatty nature of the lesion (-20/-30 HU) and showed no involvement of the sacrum surface.

MRI examination after IV contrast medium (gadobenate dimeglumine, $0.2 \mathrm{ml} / \mathrm{Kg}$ ) demon- 


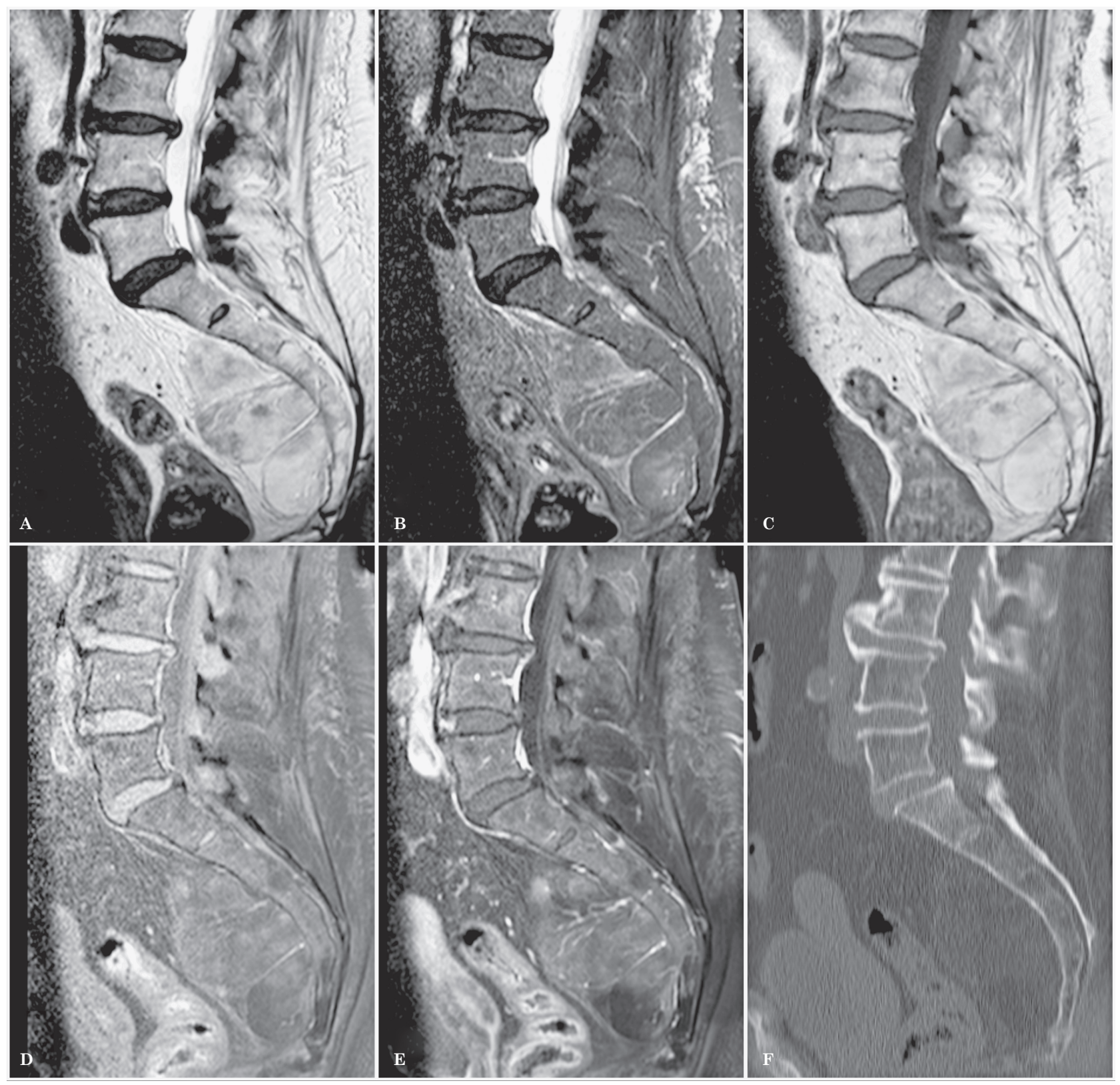

Figure 1 Sagittal MR images showing the fatty nature of the presacral mass. A) TSE-T2w (TE $100 \mathrm{~ms}$; TR 3630 ms; ETL 15, NEX 4; slice thickness $4 \mathrm{~mm}$ ); B) STIR-T2w (TE $70 \mathrm{~ms}$; TR $3000 \mathrm{~ms}$; IT $150 \mathrm{~ms}$; ETL 21, NEX 3; slice thickness $4 \mathrm{~mm}$ ); C) TSE-T1w (TE $8 \mathrm{~ms}$; TR $545 \mathrm{~ms}$; ETL 8, NEX 4; slice thickness $4 \mathrm{~mm}$ ); SPIR-T1w (TE $8 \mathrm{~ms}$; TR $574 \mathrm{~ms}$; ETL 8, NEX 4; slice thickness $4 \mathrm{~mm}$ ) before (D) and after (E) intravenous administration of paramagnetic contrast showing only a minimal enhancement of one intralesional solid tissue island. F) Sagittal $2 \mathrm{~mm}$ thick MPR reconstruction of CT examination (helical; 299mAs; 140kV; DLP $1195 \mathrm{mGy*} c \mathrm{~m}$ ) showing the fatty nature of the lesion without signs of sacral involvement.

strated only a slight enhancement of the intralesional solid nodules previously described and showed some interlobar and perilesional small vessels draining into the presacral venous plexus.

Based on the patient's age and imaging findings, an uncommon localization of extra-adre- nal myelolipoma was hypothesized even if an atypical teratoma was not excluded.

Since patient's symptoms were well correlated with CT and MR degenerative (i.e. arthrosis-related) findings, considering the patient's age, her medical history and that no cases of malignant myelolipomas have been reported 

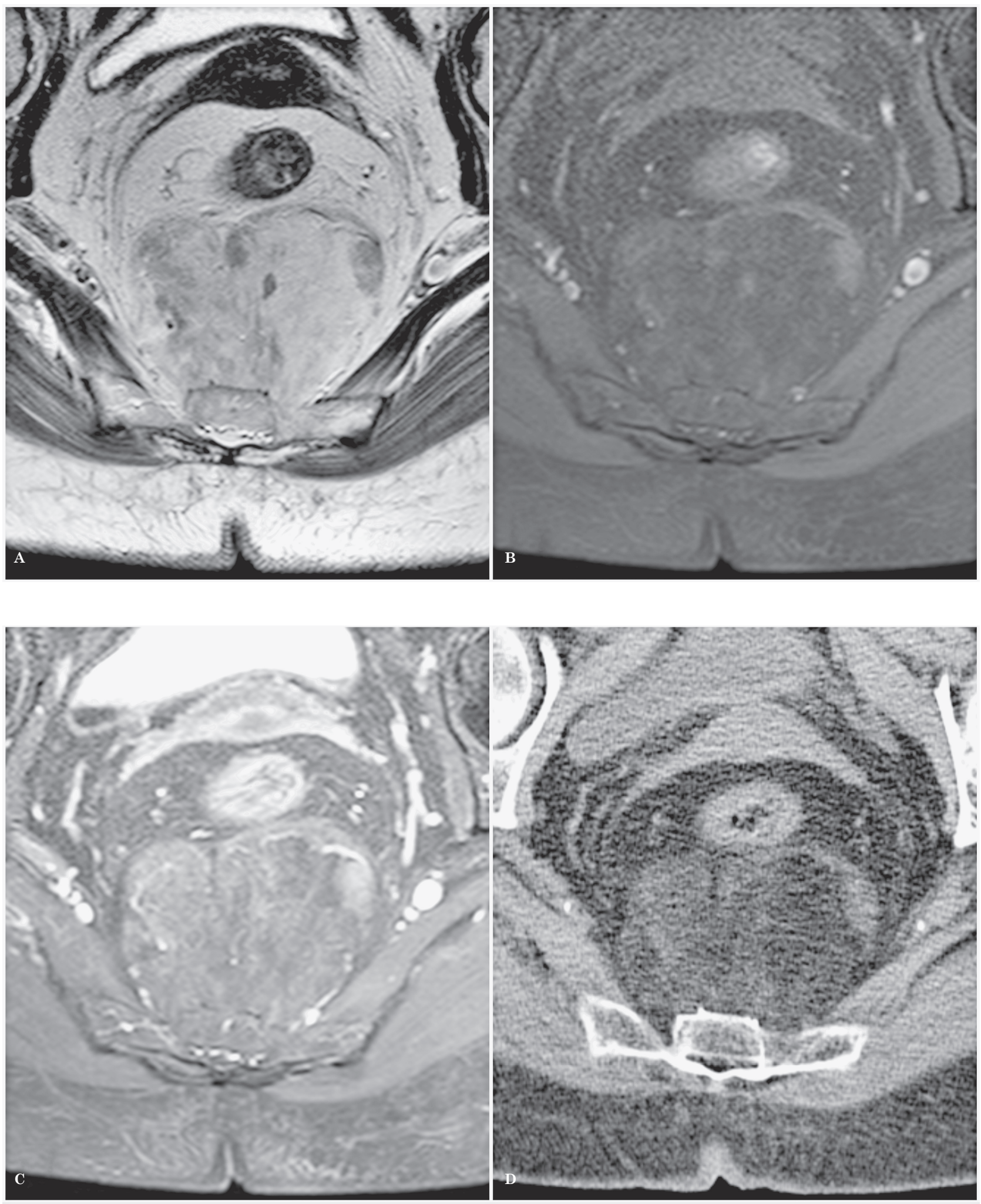

Figure 2 Axial MR images showing a few solid tissue islands into a fatty multilobated presacral mass with smooth edges and thin interlobar septa. A) TSE-T2w (TE 120 ms; TR 2340 ms; ETL 25, NEX 4; slice thickness 3 mm); SPIR-T1w (TE 8 ms; TR $561 \mathrm{~ms}$; ETL 6, NEX 4; slice thickness $3 \mathrm{~mm}$ ) before (B) and after (C) intravenous administration of paramagnetic contrast showing only a minimal enhancement of one intralesional solid tissue island. D) Oblique axial $2 \mathrm{~mm}$ thick MPR reconstruction of CT examination. 
in the English literature, no absolute indication for surgery was placed. The patient was instructed about the possibility of a haemorrhage, a common complication of typical adrenal myelolipomas. The patient later underwent to surgery in any case through an anterior transabdominal approach. Histopathologic examination of the surgical specimen confirmed the radiologic hypothesis showing a mixture characterized predominantly by mature adipocytes and haematopoietic cells including erythroid, myeloid, and megakaryocytic elements without mesenchymal tissue elements.

\section{Discussion}

Myelolipoma is a rare, well-capsulated, benign tumour that contains mature adipose tissue and various combinations of trilineage haematopoietic cells (red blood cells, white blood cells and platelets) ${ }^{1,2}$. It manifests in four distinct clinicopathologic patterns: isolated adrenal myelolipoma, adrenal myelolipoma with haemorrhage, extra-adrenal myelolipoma and myelolipoma associated with other adrenal disease ${ }^{3}$.

Although the etiology is unknown, there are several theories ${ }^{1}$ on the pathogenesis of this tumour: - metaplasia of uncommitted adrenal cortical mesenchymal cells;

- migration of haematopoietic tissue to the adrenal gland during intrauterine development.

For extra-adrenal myelolipomas there are two main pathogenetic hypotheses:

- embolization of bone marrow tissue;

- reactivation of peritoneal embryonic connective haematopoiesis tissue, in response to a triggering stimulus, like an endocrine dysfunction or in septic/infectious conditions.

Myelolipoma occurs most commonly in adrenal glands. As Baker et al. recently reported in their review, only about 50 cases of extra-adrenal myelolipomas have been reported in the literature $^{1}$. Extra-adrenal myelolipomas predominate in females $(2: 1)$ and in middle-aged to elderly patients (mean age 60 years) and vary in size (from 2 up to $26 \mathrm{~cm}$ ) ${ }^{4}$. The extra-adrenal locations include presacral and perirenal retroperitoneum, mediastinum, liver, stomach, lungs, pelvis, spleen, mesentery and muscle fascia. In some cases, extra-adrenal myelolipomas have been described in association with Cushing syndrome, Addison disease, adrenal hyperplasia and chronic exogenous steroid use ${ }^{1}$. Only one case of bilateral extra-adrenal perirenal myelolipomas has been described by Kumar et al. ${ }^{3}$.
The presacral region represents $50 \%$ of all extra-adrenal locations. The tumours are usually asymptomatic and discovered incidentally but large lesions could become symptomatic due to haemorrhage or mass effect on adjacent structures like ureters, bladder, rectum and sacral nerve roots or plexus with symptoms of urinary retention, constipation or lower extremity radiculopathy ${ }^{5}$. These lesions are typically round or oval masses, well-capsulated and adherent to the sacrum without invasion of bone or sacral nerve roots.

\section{Typical Imaging Findings}

The imaging appearance of myelolipomas varies according to tumour composition. Ultrasonography could show a hyperechoic mass (if fat predominates) or a hypoechoic mass (when there is a predominance of erythroid and myeloid cells). The variable amount of fat affects typical low attenuation CT values of the lesion that usually has some intralesional areas with higher attenuation values due to hematopoietic solid tissue islands, which may enhance after IV injection of iodine-based contrast. On MRI, fatty elements show high signal intensity (SI) on T1w and T2w sequences, which is promptly suppressed on fat-saturation pulse sequences, whereas haematopoietic tissue islands show lower signal intensity on $\mathrm{T} 1 \mathrm{w}$ and $\mathrm{T} 2 \mathrm{w}$ with variable enhancement after IV administration of paramagnetic contrast.

Several masses could be found in the presacral region incidentally or not, and they are not all benign or aggressive ${ }^{6-12}$. Differentiating presacral myelolipomas from other congenital or acquired presacral masses could be crucial for patient management.

The most widely used classification of presacral masses was proposed by Uhlig and Johnson and then modified by Dozois et al. ${ }^{6}$ who introduced a subclassification into benign and malignant tumours.

Imaging-based differential diagnosis between presacral myelolipoma and other retroperitoneal tumours containing fat could be challenging. Liposarcoma is the most common retroperitoneal neoplasia in adults (35\% of all malignant retroperitoneal soft-tissue tumours in adult patients) ${ }^{7}$. It is classified into differentiated, pleomorphic, myxoid and poorly differentiated ${ }^{8}$. Differentiated liposarcoma contains fat, but does not have a capsule, is poorly marginated, shows infiltrative growth and is 
not haemorrhagic. At CT imaging it appears as an inhomogeneous mass with areas of lowattenuating fatty components mixed with softtissue components and internal thick septations ${ }^{9,10}$. At MR imaging, it produces an image of a predominantly fatty mass with irregularly thickened or nodular septa, which decreases SI on T1W images and increases SI on T2W images ${ }^{10}$. After the administration of contrast medium, well-differentiated liposarcomas may enhance minimally or not at all.

Teratoma is a germ cell tumour (arising from totipotential cells) composed of elements from more than one germ cell layer. Its appearance ranges from cystic to solid: it may contain haematopoietic tissue, fat and calcifications but, unlike extra-adrenal myelolipoma, at microscopic examination it may also show other mesenchymal tissue elements ${ }^{1,11}$. In addition, teratoma is usually seen in younger individuals, whereas myelolipoma is typically seen in elderly patients. At CT imaging teratoma appears as a mass with fat, soft tissue, calcium and cystic attenuation; after contrast medium infusion, soft tissue components may enhance. At MRI, fat shows high SI on T1WI and T2WI; soft tissue components appear iso-hypointense in T1WI and iso-hyperintense on T2WI, calcium components usually shows very low SI on T2* gradient echo (GRE) sequences ${ }^{12}$.

Extramedullary haematopoietic tumours are typically seen in young anaemic men with hepatosplenomegaly; they are associated with myeloproliferative disorders and chronic haemolytic anaemias that result in bone marrow hyperplasia. These tumours are typically multifocal and poorly circumscribed ${ }^{1}$.

Other presacral masses difficult to differentiate from presacral myelolipomas are:

- Sacrococcygeal chordomas: these are the most frequent malignant presacral tumours that originate from the notochord, therefore they are always along the midline. They tend to invade adjacent structures and metastasize to lung, liver, lymph nodes and bone in 5$40 \%$ of cases ${ }^{12}$. Conventional spine $\mathrm{x}$-ray could demonstrate an aspecific osteolytic lesion. At CT they appear as an inhomogeneous, wellcircumscribed mass with frequent peripheral calcifications; vertebral bodies are usually infiltrated/destroyed by the lesion. At MRI this lesion appears hypo- to isointense (compared to marrow) on $\mathrm{T} 1 \mathrm{~W}$ images and hyperintense to cerebrospinal fluid (CSF) on T2W images. MRI could be helpful demonstrating relationships with adjacent structures like meninges, vessels and especially nerves ${ }^{12}$. Sacrococcygeal chordomas show variable enhancement after IV administration of paramagnetic contrast.

- Dermoid and epidermoid cysts: they result from an abnormal closure of the ectodermal tube so they are typically along the midline too. Dermoids are composed of stratified squamous epithelium and skin appendages like sweat glands, hair follicles or sebaceous cysts. Epidermoids are characterized only by the presence of squamous epithelium. Dermoids usually show a striking fat density on CT and may contain capsular calcifications; on MRI they usually show a heterogeneous hyperintense signal on T1WI and T2WI, a low signal on fat suppressed sequences and they are less likely to show restricted diffusion than epidermoids on diffusion weighted imaging (DWI). Epidermoids usually resemble CSF on both CT and MR examinations, may contain capsular calcifications and they usually show a CSF-like signal on conventional pulse sequences with a typical restriction of diffusion on DWI sequences. After administration of contrast medium they usually show a minimal marginal enhancement or none at all ${ }^{12}$.

- Anterior sacral meningocele: is a herniation of the dural sac through a defect in the anterior sacrum wall. The dural sac is in continuity with the dural space and contains CSF and spinal roots ${ }^{11}$. MRI shows a non-enhancing pelvic mass with CSF-like intensity that may present internal septa; cord tethering and/or lipomas and dermoids could coexist ${ }^{12}$.

- Presacral pelvic retroperitoneal schwannoma: this is a benign, slow-growing encapsulated tumour of nerve sheath origin. At CT imaging, it manifests as a mild hypo-attenuating mass along the expected course of the nerves; calcifications, cystic degeneration or haemorrhage may also be seen. At MRI the schwannoma is iso/hypointense on T1WI (relative to cord and roots) and hyperintense on T2WI in $75 \%$ of cases. Usually it has an intense and homogeneous enhancement of solid components after contrast medium infusion; $90 \%$ are solitary and sporadic while the others are neurofibromatosis (NF)-associated or multiple but unassociated with $\mathrm{NF}^{13}$. Rarely a malignant degeneration into malignant sarcomas has been described in NF syndromes ${ }^{14}$.

Although imaging characteristics of these lesions allow a presumptive diagnosis, core or needle biopsy is often required. However many surgeons consider presacral biopsy a contraindication in any resectable lesion due 
to the risks of seeding along the biopsy needle tract $^{15}$. Therefore surgical resection is required and can be performed by three different approaches: anterior or transabdominal, posterior or perineal and combined abdominoperineal approach ${ }^{15}$. The patient's outcome depends on the nature and location of the lesion. The clinical history and imaging play a predominant

\section{References}

1 Baker KS, Lee D, Huang M, et al. Presacral myelolipoma: a case report and review of imaging findings. $J$ Radiol Case Rep. 2012; 6 (6): 1-9.

2 Saboorian MH, Timmerman TG, Ashfaq R, et al. Fineneedle aspiration of a presacral myelolipoma: a case presentation with flow cytometry and immunohistochemical studies. Diagn Cytopathol. 1999; 20 (1): 47-51.

3 Kumar M, Duerinckx AJ. Bilateral extraadrenal perirenal myelolipomas: an imaging challenge. Am J Roentgenol. 2004; 183 (3): 833-836. doi: 10.2214/ ajr.183.3.1830833.

4 Kammen BF, Elder DE, Fraker DL, et al. Extraadrenal myelolipoma: MR imaging findings. Am J Roentgenol. 1998; 171 (3): 721-723. doi: 10.2214/ajr.171.3.9725304

5 Singla AK, Kechejian G, Lopez MJ. Giant presacral myelolipoma. Am Surg Apr. 2003; 69: 334-338.

6 Hassan I, Wietfeldt ED. Presacral tumors: diagnosis and management. Clin Colon Rectal Surg. 2009; 22 (2): 84-93. doi: 10.1055/s-0029-1223839.

7 Craig WD, Fanburg-Smith JC, Henry LR, et al. Fatcontaining lesions of the retroperitoneum: radiologicpathologic correlation. Radiographics. 2009; 29 (1) 261-290. doi: 10.1148/rg.291085203.

8 Nishino M, Hayakawa K, Minami M, et al. Primary retroperitoneal neoplasms: CT and MR imaging findings with anatomic and pathologic diagnostic clues. RadioGraphics. 2003; 23: 45-47. doi: 10.1148/rg.231025037.

9 Coleman BG, Mulhern CB, Arger PH, et al. New observations of soft tissue sarcomas with contrast medium enhanced computed tomography. J Comput Tomogr. 1985; 9 (3): 187-193. doi: 10.1016/0149-936X(85)90059-1.

10 Jelinek JS, Kransdorf MJ, Shmookler BM, et al. Liposarcoma of the extremities: MR and CT findings in the histologic subtypes. Radiology. 1993; 186: 443-449. doi: 10.1148/radiology.186.2.8421750.

11 Alampady K. Shanbhogue MD, Najla Fasih FRCR, et al. Uncommon Primary Pelvic Retroperitoneal Masses in Adults: A Pattern-based Imaging Approach. RadioGraphics. 2012; 32: 795-817. doi: 10.1148/rg.323115020.

12 Ross JS, Moore KR, Borg B, et al. Diagnostic Imaging: Spine. Salt Lake City, Utah: Amirsys Inc. 2010.

13 Louis DN, Ohgaki H, Wiestler OD, Cavenee WK. (Eds.): WHO Classification of Tumours of the Central Nervous System. Lyon: IARC. 2007. role, with particular reference to MRI in the diagnosis of presacral masses. Conventional pulse sequences with and without fat saturation findings are often sufficient to rule out a diagnostic hypothesis. DWI sequences, more recently introduced for spine studies, may represent an added value for the differential diagnosis of some of these presacral masses.
14 Levy AD, Patel N, Dow N, et al. From the archives of the AFIP: abdominal neoplasms in patients with neurofibromatosis type 1: radiologic-pathologic correlation. Radiographics. 2005; 25 (2): 455-480. doi: 10.1148/ rg. 252045176.

15 Hassan I, Wietfeldt ED. Presacral Tumors: Diagnosis and Management. Clin Colon Rectal Sur May. 2009; 22 (2): 84-93. doi: 10.1055/s-0029-1223839.
Dr Cesare Gagliardo

Department of Biopathology

and Medical and Forensic Biotechnologies Section of Radiological Sciences

University of Palermo

Via del Vespro n. 129

90127 Palermo, Italy

Tel: +39-091-6552361

Fax: +39-091-6552324

E-mail: cesare.gagliardo@unipa.it cesare.gagliardo@gmail.com 